\title{
Single-Atom Density of States of an Optical Lattice
}

\author{
Chris Hooley and Jorge Quintanilla \\ School of Physics and Astronomy, University of Birmingham, Edgbaston, Birmingham B15 2TT, United Kingdom
}

(Received 11 December 2003; published 19 August 2004)

\begin{abstract}
We consider a single atom in an optical lattice, subject to a harmonic trapping potential. The problem is treated in the tight-binding approximation, with an extra parameter $\kappa$ denoting the strength of the harmonic trap. It is shown that the $\kappa \rightarrow 0$ limit of this problem is singular, in the sense that the density of states for a very shallow trap $(\kappa \rightarrow 0)$ is qualitatively different from that of a translationally invariant lattice $(\kappa=0)$. The physics of this difference is discussed, and densities of states and wave functions are exhibited and explained.
\end{abstract}

DOI: $10.1103 /$ PhysRevLett.93.080404

PACS numbers: $03.75 . \mathrm{Lm}, 03.65 . \mathrm{Sq}, 39.25 .+\mathrm{k}$

The achievement of degeneracy in atomic gases has led to a surge of activity. One reason is that these systems provide a test bed for theories developed in the context of solid-state physics. Indeed there have been experimental observations of Bose-Einstein condensation (BEC) [1], vortex lattices [2,3], superfluid-insulator transitions [4], fermion degeneracy [5], and, very recently, advances have been made towards Bardeen-Cooper-Schrieffer (BCS) superfluidity [6]. A great advantage of these systems is one's unprecedented control of the experimental situation. By varying the magnetic field strength, particle-particle interactions can be altered. Moreover, one can use lasers to create an optical lattice whose parameters can be changed at will [7]. The latter scenario has been the subject of recent theoretical attention [8-11].

One key difference between these systems and the usual models of the solid state is that they are not translationally invariant, because of the trapping potential necessary to confine the gas. However, in the usual setup, when the trap is the only external potential, the local density approximation can be employed, and thermodynamic quantities can be simply related to their values in the absence of the trap [12]. Trapped atoms are, therefore, a good model of the continuum many-particle system analogous, in the fermion case, to "jellium," the ideal metal of many-electron theory. One thus expects that, when the periodic optical lattice potential is added, an experimental model of electrons in real crystals can be obtained.

In this Letter we take a first step towards testing this expectation by obtaining the single-particle density of states (DOS). This is the starting point for understanding many key features of degenerate quantum systems. For example, in an ideal Bose gas it determines whether there is BEC [13], while in a weakly attractive Fermi gas it gives the critical temperature for BCS superfluidity [14]. The DOS becomes a smooth curve only in the limit in which the trap is very weak so that the spectrum becomes a continuum. As we shall see, this limit is singular, in the sense that the DOS is qualitatively different from that of an infinite, translationally invariant lattice.
Model.-We consider a single particle, with no internal degrees of freedom, moving in an infinite tight-binding lattice with one orbital per site and hopping between nearest neighbors. The particle is confined to a finite region by a harmonic trapping potential. For simplicity, we assume a $d$-dimensional hypercubic lattice. For $d>1$, the problem is separable, so we shall focus our attention on the 1D case, indicating how the results for $d \geqslant 2$ follow as the need arises. The Hamiltonian for $d=1$ is

$$
H=-t \sum_{j}(|j\rangle\langle j+1|+\text { H.c. })+\frac{1}{2} \kappa \sum_{j}(a j)^{2}|j\rangle\langle j|,
$$

where $j$ is a site label, $a$ the lattice constant, $\kappa$ the strength of the trap, and $t$ the nearest-neighbor hopping integral. (This model also occurs in the related subject of the dynamical diffraction of atoms by static light fields; see, for example, Ref. [15] and references therein.) Note that the trap is centered at a lattice site; see Refs. $[16,17]$ for a discussion of the nontrivial effect of incommensuration. The model can be generalized also by considering anisotropic lattices and trapping potentials.

Before describing the properties of the model, let us discuss its validity. In experiments [7], the lattice is generated by counter-propagating laser fields, giving rise to a static, periodic potential [18] of the form $U(x)=$ $-U_{0}\left[\cos \frac{2 \pi x}{a}+1\right]$. Equation (1) is valid when the wells of this potential are sufficiently deep: $U_{0} \gg \hbar^{2} / m a^{2}$, where $m$ is the mass of one atom. In this intense-laser regime, one can approximate the orbitals at the bottom of each well by those of a harmonic oscillator, and the usual method [19] yields the tight-binding parameter as [9] $t=$ $U_{0} \exp \left[-\frac{\pi}{2} \sqrt{U_{0}\left(\hbar^{2} / m a^{2}\right)^{-1}}\right]$, which is much smaller than the first excitation energy of the orbital. The effect of the overall trapping potential on $t$ can be neglected provided that the potential energy difference between the minima of neighboring wells, $\Delta E_{j}$, is much less than the barrier height, $U_{0}$. Now, $\Delta E_{j} \approx \kappa a^{2} j$ for $j \gg 1$; hence our model is accurate for states whose wave functions do not extend beyond site $j_{c} \equiv U_{0} / \kappa a^{2}$. Hence the state's energy $\epsilon$ must satisfy $\epsilon \ll \kappa a^{2} j_{c}^{2}$, i.e., $\epsilon \ll \epsilon_{\max } \equiv U_{0}^{2} / \kappa a^{2}$. We would 
therefore like to be sure that $\epsilon_{\max } \gg t$, which is true provided that $U_{0} \gg \kappa a^{2}$.

We are interested in the DOS, $\rho(\epsilon) \equiv \sum_{\nu} \delta\left(\epsilon-\epsilon_{\nu}\right)$, which is a smooth curve only in the limit in which the spectrum $\left\{\epsilon_{\nu}\right\}$ is a continuum. Let us discuss some limiting behaviors of $\rho(\epsilon)$. The solution is well known for $\kappa=$ 0 , when the lattice forms a large "box" of length $L$. In this case one obtains Bloch waves with the band dispersion relation $\epsilon_{k}=-2 t \cos k a$, where $k$ is the wave number. When $L \gg a$, $k a$ runs continuously from $-\pi$ to $\pi$. In this limit, $\rho(\epsilon)$ is nonzero only for $|\epsilon| \leqslant 2 t$ (this region we call the "band"), and has square-root singularities at $\epsilon=$ $\pm 2 t$ (the "top" and "bottom" of the band). For $d=2$, the DOS extends from $\epsilon=-4 t$ to $4 t$ and is finite at these points, but exhibits a "van Hove" logarithmic singularity at $\epsilon=0$ [19].

In our model, the lattice itself is taken to be infinite; the finite extent of the wave functions is determined instead by the strength, $\kappa$, of the harmonic trap. The corresponding length scale is [12] $l \equiv \sqrt{t / \kappa}$. This suggests that, in this model, "continuum limit" should be taken to mean $l \gg a$ or, equivalently, $\kappa a^{2} \ll t$. We shall work in this limit henceforth (unless otherwise stated).

Let us consider the low- and high-energy states of (1). For energies near the bottom of the band, the dispersion relation has the free-particle form $\epsilon_{k} \approx-2 t+\hbar^{2} k^{2} / 2 m^{*}$, where $m^{*}=\hbar^{2} / 2 t a^{2}$. Thus we would expect the low-lying eigenstates of (1) to resemble those of the usual continuum harmonic oscillator. Indeed, one can show explicitly that $\left|\phi_{0}\right\rangle=N_{0} \sum_{j} e^{-a j^{2} / 2 \sqrt{2} l}|j\rangle$ is the ground state to second order in $a / l$. Its energy is $\epsilon_{0}=-2 t+\hbar \omega^{*} / 2+$ $o\left([a / l]^{2}\right)$, where $\omega^{*} \equiv \sqrt{\kappa / m^{*}}$. To first order in $a / l$, this equals the usual result for the harmonic oscillator, measured from $\epsilon=-2 t$. The low-lying states are obtained similarly. Since the DOS of the 1D harmonic oscillator is constant and equal to $1 / \hbar \omega^{*}$, we conclude that the lowenergy limit of the DOS of (1) is given by $\rho(\epsilon) \rightarrow 1 / \hbar \omega^{*}$ as $\epsilon \rightarrow-2 t$ for $d=1$. For $d=2$, the DOS of the harmonic oscillator is $\rho(\epsilon) \propto \epsilon$, so we expect a vanishing density of states as $\epsilon \rightarrow-4 t$ in this case.

For high energies, the physical nature of the states is quite different. When $\epsilon \gg t$, the first term in (1) can be neglected, and thus the eigenstates of the Hamiltonian are the position eigenstates $|j\rangle$, with energies $\epsilon_{j}=$ $\kappa a^{2} j^{2} / 2$. The high-energy 1D DOS is then easily shown to be $\rho(\epsilon \gg t)=\sqrt{2 / \kappa a^{2}} \epsilon^{-1 / 2}$. In $2 \mathrm{D}$, the analogous calculation gives $\rho(\epsilon) \rightarrow 2 \pi / \kappa a^{2}$ as $\epsilon / t \rightarrow \infty$.

$W K B$ analysis.-To extend our treatment of (1) to all energies, we employ the Wentzel-Kramers-Brillouin (WKB) approximation [20]; it has already been applied to the momentum-space version of the problem in Ref. [21]. By contrast, our WKB-type analysis will be performed in real space [15]. It differs from traditional WKB in that $\hbar$ does not appear anywhere in (1); instead we choose $a$ as our small parameter. As in the ordinary WKB method, we write the wave function in the form $\psi(x) \sim \exp \left[i \int^{x} k\left(x^{\prime}\right) d x^{\prime}\right]$. The energy is determined by the usual quantization condition, $\oint k(x) d x=2 \pi(n+\gamma)$, where $n$ is an integer and $\gamma$ a constant. Differentiating this, we obtain the DOS: $\rho(\epsilon) \equiv d n / d \epsilon=\frac{1}{2 \pi} \frac{\partial}{\partial \epsilon} \oint k(x) d x$ (note that $\gamma$ is not required).

On the other hand, the orbit equation is modified by replacing the free-particle dispersion by the tight-binding form [22]: $-2 t \cos (k a)+\frac{1}{2} \kappa x^{2}=\epsilon$. The local wave number becomes $k(x)= \pm \frac{1}{a} \arccos \left(\frac{\kappa x^{2}-2 \epsilon}{4 t}\right)$. This makes a significant difference, because it introduces two new turning points. The classical turning points (where $k=0$ ) still exist, and are found at $x= \pm x_{c}$, where

$$
x_{c}=\sqrt{\frac{2 \epsilon+4 t}{\kappa}}=2 l \sqrt{\frac{\epsilon}{2 t}+1} ;
$$

now, however, two new points appear for energies $\epsilon>2 t$. They are associated with Bragg reflection, and they occur at $x= \pm x_{b}$, where

$$
x_{b}=\sqrt{\frac{2 \epsilon-4 t}{\kappa}}=2 l \sqrt{\frac{\epsilon}{2 t}-1} .
$$

We shall refer to them as "Bragg turning points," and the region between them as "Bragg forbidden."

The quantization condition now becomes more subtle. To see why, note that in the Bragg forbidden region $k(x) a=\pi+i \operatorname{arccosh}\left(\frac{2 \epsilon-\kappa x^{2}}{4 t}\right)$ : the wave function continues to oscillate for $|x|<x_{b}$, despite the damping of its amplitude. The quantization condition must therefore include these oscillations; it becomes:

$$
2 \int_{x_{b}}^{x_{c}} k(x) d x+\frac{2 \pi x_{b}}{a}=\pi(n+\gamma) .
$$

Now we can obtain the DOS by differentiating (4), remembering that $x_{b}$ and $x_{c}$ depend on $\epsilon$ according to (2),(3); we obtain a lengthy analytic expression for $\rho(\epsilon)$. It is plotted in Fig. 3(a), along with the DOS obtained from numerical diagonalization of (1), with appropriate provision for finite-size scaling (see below): clearly the agreement is excellent. This was foreseeable, since aside from a vanishing number of low-lying states, all the wave functions have slowly varying $k(x)$ (or, in the Bragg forbidden region, not varying at all); thus the WKB method is expected to be accurate at all energies. Note that $\rho(\epsilon)$ is qualitatively different from the DOS of the $\kappa=0$ tight-binding model, while it agrees quantitatively with $\rho \rightarrow 1 / \hbar \omega^{*}$ at $\epsilon=-2 t$ and with $\rho \rightarrow \sqrt{2 / \kappa a^{2}} \epsilon^{-1 / 2}$ for $\epsilon / t \gg 1$, the limiting expressions obtained above.

Calculating the density of states for $d>1$ is simple, because the Hamiltonian is separable. Consider, for example, the case of $d=2$. It is not hard to show that $\rho_{2 \mathrm{D}}(\epsilon)=\int_{-\infty}^{\epsilon} \rho(\epsilon-\chi) \rho(\chi) d \chi$, where $\rho_{2 \mathrm{D}}$ is the DOS of the 2D system, and $\rho$ is the DOS of the 1D Hamiltonian (1). The result of applying this formula is presented in Fig. 3(b), and compared with numerical 
results; again, the agreement is excellent. Note that the steep feature at $\epsilon=0$ and the kink at $\epsilon=4 t$ both result from the logarithmic singularity in the 1D DOS. As $\epsilon \rightarrow$ $-4 t$, we recover $\rho_{2 \mathrm{D}} \rightarrow 0$, as expected from the analytic arguments above. Furthermore, the large- $\epsilon$ asymptote is exactly the constant predicted by that analysis. Higherdimensional densities of states may be generated in a similar way.

Finite-size scaling: Numerics.-In the foregoing we obtained the DOS in the continuum limit $l / a \rightarrow \infty$. However, in experiments, $l / a$ is finite. We should therefore check that our results resemble the behavior of the system when it has a steeper trap. Thus we have studied the single-particle spectrum and wave functions by numerical diagonalization of (1) [23]. By considering increasingly shallow traps, the numerical calculations also allow us to confirm the validity of WKB for the continuum limit. We have applied the same method in $d=2$, starting with the 2D version of (1). The results are analogous so we shall, as above, describe in detail the 1D case only.

In order to have a finite-dimensional Hamiltonian matrix, we must describe the lattice by a finite number of sites, $N$. This leads to numerical artefacts for $\epsilon>\epsilon_{N}$, defined by $N \equiv 2 x_{c}\left(\epsilon_{N}\right) / a$, as the particle visits the artificial limits of the lattice. On the other hand, we expect this to be essentially equivalent to the original problem for $\epsilon \ll \epsilon_{N}$. Calculations for increasingly large $N$ confirm this (see the inset of Fig. 2).

Figure 1 shows some numerically determined wave functions for fixed trap strength. In spite of the relatively small system size $(l / a=10)$, the states seem well described by the continuum-limit solution. At low energies, $\epsilon \lesssim 0$, the wave functions resemble those of a continuum 1D harmonic oscillator. At higher energies, the period of these oscillations starts to become similar to the lattice constant, and commensuration effects emerge. At $\epsilon=2 t$,

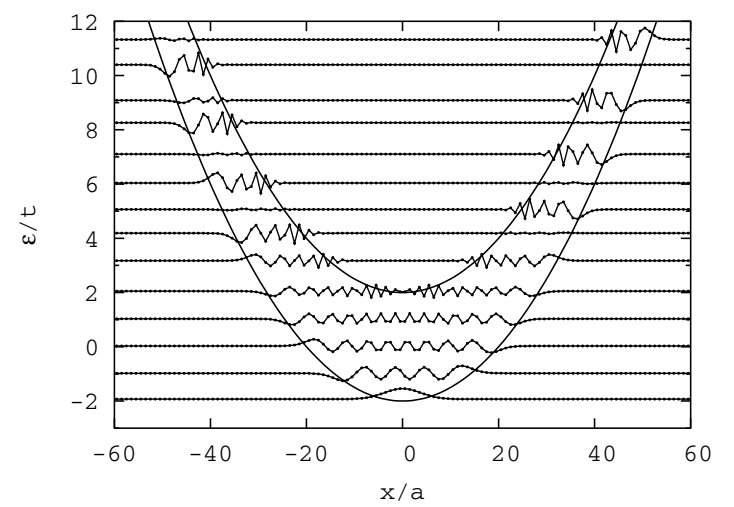

FIG. 1. Numerically determined wave functions for a trap with $\kappa a^{2}=t / 100$. Each wave function has been offset along the $y$-axis by its energy, in units of the hopping integral. The numerical calculation uses a lattice with $N=130$ sites. For the energies considered, there are no noticeable numerical artefacts. The lower and upper solid curves correspond to the classical and Bragg turning points, Eqs. (2),(3). as that period reaches its minimum, the Bragg reflection points appear. Immediately above the top of the band, $\epsilon \gtrsim 2 t$, the modulation of the wave number is evident, reaching its minimum and maximum near the classical and Bragg turning points, respectively. The rapid oscillations continue in the Bragg forbidden region, where the amplitude decays exponentially. As the energy rises further, the distance between the Bragg and classical turning points shrinks so that fewer and fewer oscillations take place between these two points. Eventually the particle is forced to localize on single sites (not shown). Note that the Bragg and classical turning points are very accurately described by Eqs. (2),(3), even for the relatively small value of $l / a$ considered here. Furthermore, the number of high-energy (localized) solutions is reproduced correctly. This is because the condition that the wave function's phase be single-valued reduces, at $k=\pi$, to the condition that the localized states be separated by an integer number of lattice spacings $(x=n a)$.

We now turn to the DOS. Obviously for finite $l / a$ the spectrum is not a continuum so the DOS is not a smooth curve. However, one can smooth it by the following procedure: we divide an energy range into intervals, of width $\Delta \epsilon$, and count the states within each interval. We then rescale the vertical axis of the resulting histogram so that each column represents the number of states per unit energy, on average, in the corresponding interval. The result is a "binned" DOS that looks smooth only if the intervals are sufficiently wide. Figure 2 shows the result for different values of $\kappa a^{2} / t$. Note that we have further rescaled the vertical axis by an overall factor of $a / l$. This leads to the collapse of all the data onto a single curve, suggesting that the continuum-limit DOS describes the

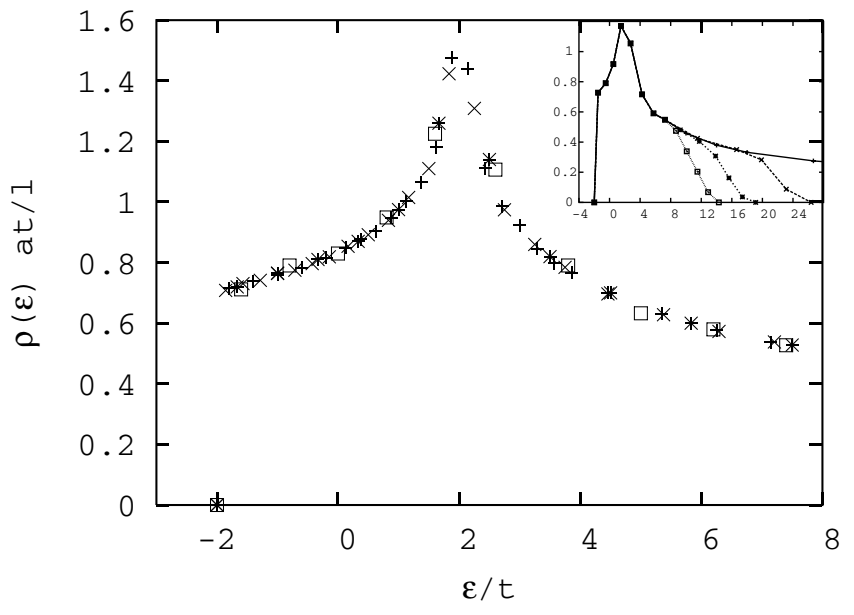

FIG. 2. Finite-size scaling of the binned DOS for 1D lattices with finite strength of the trapping potential: $\kappa a^{2} / t=$ $5 \cdot 10^{-6}(+), 10^{-5}(\times), 10^{-4}(*)$, and $10^{-3}(\square)$. In all cases the numerical cutoff energy, $\epsilon_{N}$, lies outside the energy range showed in the plot. This is illustrated in the inset: it shows the DOS for fixed $\kappa a^{2}=t / 1000$, but calculated using lattices with different numbers of sites: $N=300(\square), 350(*), 420(\times)$, and $2000(+)$. 

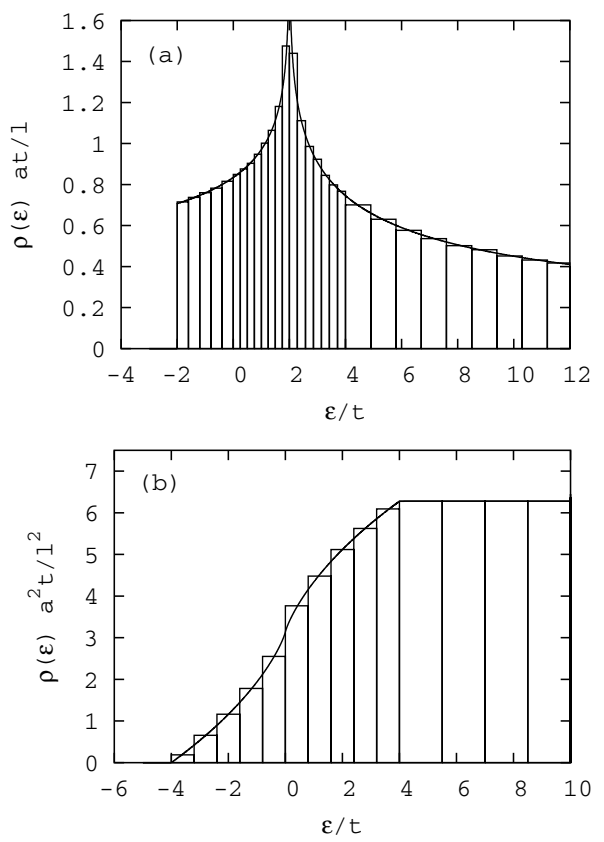

FIG. 3. DOS of a single atom in an optical lattice for (a) $d=1$ and (b) $d=2$. Curves: continuum-limit results obtained within the WKB approximation. Histograms: numerical diagonalization for finite-size systems with trap strengths $\kappa=5 \times 10^{-6} t / a^{2}$ (a) and $\kappa=1.5 \times 10^{-2} t / a^{2}(\mathrm{~b})$, using lattices with $N=5000$ and 10000 sites, respectively.

overall distribution of energy levels even for quite steep traps, with only $l / a \sim 100$ sites. The inset illustrates the numerical cutoff artefact mentioned above. Finally, Fig. 3 compares the DOS of two finite (but fairly large) systems to the WKB predictions obtained above in $1 \mathrm{D}$ and $2 \mathrm{D}$, respectively. Evidently our WKB approach captures the continuum limit rather well.

Conclusion.-We have calculated the DOS for a single atom in an optical lattice; this should be regarded as the logical first step towards a detailed theory of the experimentally realized many-particle systems. Our results are based on WKB theory, and refer to the limit $l \gg a$ or, equivalently, when the trapping potential becomes flat: $\kappa \rightarrow 0$. Numerical diagonalization reveals this theory to be extremely accurate in that case, and moreover shows that, for finite-size systems, the binned DOS has the same overall features. Our main result is that the DOS, in this limit, is radically different from what is obtained for a homogeneous lattice (i.e., for $\kappa=0$ rather than $\kappa \rightarrow 0$ ): the square-root singularities in the $1 \mathrm{D}$ case are replaced by a logarithmic one, and the logarithmic van Hove singularity in 2D disappears altogether. Moreover, our theory provides a detailed picture of how this comes about. The crucial features are the new turning points, associated with Bragg reflection, that appear at energies above the top of the conduction band. The possibility of inducing Bragg reflection by using a time-dependent external potential was considered in [11]. We have shown that Bragg reflection is, in fact, essential to understand the equilibrium single-particle spectrum of the optical lattice.

We thank J. M. F. Gunn, A. F. Ho, M.W. Long, and A. J. Schofield for useful discussions. We acknowledge financial support from EPSRC $(\mathrm{CH})$ and the Leverhulme Trust (JQ).

Note added.-It has been drawn to our attention that some of the features discussed here have recently been pointed out in an exact diagonalization study [25].

[1] M. H. Anderson, J. R. Ensher, M. R. Matthews, C. E. Wieman, and E. A. Cornell, Science 269, 198 (1995).

[2] J. R. Abo-Shaeer, C. Raman, J. M. Vogels, and W. Ketterle, Science 292, 476 (2001).

[3] P.C. Haljan, I. Coddington, P. Engels, and E. A. Cornwell, Phys. Rev. Lett. 87, 210403 (2001).

[4] M. Greiner, O. Mandel, T. Esslinger, T.W. Hänsch, and I. Bloch, Nature (London) 415, 39 (2002).

[5] B. DeMarco and D. Jin, Science 285, 1703 (1999).

[6] M. Greiner et al., cond-mat/0311172.

[7] M. Greiner, I. Bloch, O. Mandel, T.W. Hänsch, and T. Esslinger, Phys. Rev. Lett. 87, 160405 (2001).

[8] R. G. Scott, S. Bujkiewicz, T. M. Fromhold, P. B. Wilkinson, and F.W. Sheard, Phys. Rev. A 66, 023407 (2002).

[9] H. Büchler, G. Blatter, and W. Zwerger, Phys. Rev. Lett. 90, 130401 (2003).

[10] A. Ho, M. Cazalilla, and T. Giamarchi, Phys. Rev. Lett. 92, 13045 (2004).

[11] R. Scott, A. Martin, T. Fromhold, S. Bujkiewicz, F. Sheard, and M. Leadbeater, Phys. Rev. Lett. 90, 110404 (2003).

[12] K. Damle, T. Senthil, S. Majumdar, and S. Sachdev, Europhys. Lett. 36, 7 (1996).

[13] P. Nozieres, in Bose-Einstein Condensation, edited by A. Griffin, D.W. Snorke, and S. Stringari (Cambridge University Press, Cambridge, England, 1995).

[14] P. G. de Gennes, Superconductivity of Metals and Alloys (Benjamin, New York, 1966).

[15] D. H. O’Dell, J. Phys. A 34, 3897 (2001).

[16] E. Chalbaud, J.-P. Gallinar, and G. Mata, J. Phys. A 19, L385 (1986).

[17] J. Gallinar and E. Chalbaud, Phys. Rev. B 43, 2322 (1991).

[18] M. B. Dahan, E. Peik, J. Reichel, Y. Castin, and C. Salomon, Phys. Rev. Lett. 76, 4508 (1996).

[19] N.W. Ashcroft and N. D. Mermin, Solid State Physics (Thomson Learning, New York, 1976).

[20] C. M. Bender and S. A. Orszag, Advanced Mathematical Methods for Scientists and Engineers (Springer, New York, 1999).

[21] A. Polkovnikov, S. Sachdev, and S. M. Girvin, Phys. Rev. A 66, 053607 (2002).

[22] I. Lifshits, M. Azbel, and M. Kaganov, Electron Theory of Metals (Consultants Bureau, New York, 1973).

[23] We used the diagonalization function of [24].

[24] J.W. Eaton et al., Octave, available under the General Public License at http://www.octave.org.

[25] M. Rigol and A. Muramatsu, cond-mat/0311444. 